\title{
ON K-LINE CENTRAL REVERSALS
}

\author{
(Research Note)
}

JAY M. PASACHOFF and HAROLD ZIRIN

Big Bear Solar Observatory, Hale Observatories, California Institute of Technology, Carnegie Institute of Washington, Pasadena, Calif. 91109, U.S.A.

(Received 11 February, 1971)

The double reversal at the center of the spatially-averaged profiles of the $\mathrm{H}$ and $\mathrm{K}$ resonance lines in the solar atmosphere has been used as a probe to give the distribution of temperature and density with height. Details of the interpretations depend on the mechanism assumed for the formation of the central reversals. The non-LTE theory of stellar atmospheres has succeeded in explaining a symmetric double reversal; the history of this effort has recently been reviewed by Linsky and Avrett (1970).

Zirin (1966) pointed out that with the best resolution, single-peaked asymmetric structures often occur. Pasachoff (1970) studied these single peaks quantitatively, and pointed out that when single peaks are detectable and the seeing is good, then most of the fine-structure profiles on those frames show single-peaked profiles, i.e. that regions containing mostly single-peaked profiles exist. Wilson and Evans (1971) have studied a time sequence of frames, and agree that this is a basic type of structure to be explained. But they point out many double-peaked profiles as well.

It has long seemed that both types of profiles existed. However, it is not at all clear from which features on the Sun these different profiles come. There is a qualitative distinction, for example, among profiles taken over plages, over the supergranulation network, and in the center of supergranulation cells.

None of the observers so far have specified in what type of chromospheric region (the terms 'quiet' or 'active' are insufficient) their spectra were taken. Without this information a detailed discussion is impossible. Seeing fluctuations from frame to frame on sequences of $\mathrm{K}$-line spectra further complicate the resolution of this question, which must await simultaneous high-resolution spectra and filtergrams.

Wilson and Evans ask for confirmation of their result that velocities of the order of $50 \mathrm{~km} / \mathrm{sec}$ exist at the levels at which the $\mathrm{K}_{2}$ peaks are formed. Pasachoff $e t$ al. (1968) found disturbances propagating in the chromosphere in spicules exceeding hundreds of kilometers/sec, and which must therefore arise from Alfvén waves. They also reported horizontal motions in spicules, parallel to the solar surface. Weart (1970) has further studied these horizontal motions, which could correspond to those seen on the disc by Wilson and Evans.

The question of interest is how to fit theoretical models to the observational data. The 'standard' non-LTE model has great difficulty explaining any singly-peaked profiles. The static calculations that are the current state of the art give symmetric 
profiles, and it is commonly assumed that asymmetries, such as the slightly greater violet peak measured on average profiles, can be explained by a slight outward motion of the emitting regions on the solar surface. One point in favor of this explanation is that it seems reasonable for the material to be moving outward. However, the solar K-line fine structure can be considered as an example of the structure on more distant stars (Pasachoff, 1971). Some stars, such a $\alpha$ Bootis (Arcturus) where the average $K_{2}$ red peak is substantially brighter than the average $K_{2}$ violet peak, are more difficult to explain with the 'standard' theory.

Wilson and Evans show an example of a single $\mathrm{K}_{2}$ peak that appears, sequentially, in opposite wings of the line. Assuming that this is real, rather than a statistical effect of overlapping but different features, recalls the spicular oscillation discussed by Pasachoff et al. (1968). It was there suggested that the central reversal visible in limb observations arose from an absorbing interspicular medium, because the reversal remained unshifted. On the disc, Pasachoff (1970) reported shifting of the central reversal in the direction opposite to the $\mathrm{K}_{2}$ peak, but the appearance of peaks on red and violet sides sequentially without appearing at the line center indicates that there is at least some contribution from absorbing material to the formation of $\mathrm{K}_{3}$, and that $\mathrm{K}_{3}$ must be partially formed at a higher chromospheric level than the peaks. Some $\mathrm{K}_{3}$ absorption had to be invoked in any case as a possible explanation for the limb behavior of emission peaks. Doubly-peaked profiles may still be formed at least partly by the 'standard' non-LTE method, arising as a consequence of the existence of a chromosphere (Jefferies, 1968). But we must know the details of the chromospheric structure in which they arise.

\section{Acknowledgements}

This work was partially supported by NASA Grant NGR 05-002-034 and by NSF Grant No. GA 24015.

\section{References}

Jefferies, J. T.: 1968, Spectral Line Formation, Blaisdell, Waltham, Mass.

Linsky, J. L. and Avrett, E. H.: 1970, Publ. Astron. Soc. Pacific 82, 169.

Pasachoff, J. M.: 1970, Solar Phys. 12, 202.

Pasachoff, J. M.: 1971, Astrophys. J. 164 (in press).

Pasachoff, J. M., Noyes, R. W., and Beckers, J. M.: 1968, Solar Phys. 5, 131.

Weart, S. R.: 1970, Solar Phys. 13, 310.

Zirin, H.: 1966, The Solar Atmosphere, Blaisdell, Waltham, Mass. 